\title{
Impact of Comorbidities On Physical Function And Survival of Middle-Aged As Compared To Older, Individuals With Cancer.
}

Bogda Koczwara ( $\sim$ bogda.koczwara@flinders.edu.au )

The Flinders University of South Australia https://orcid.org/0000-0002-1201-1642

\section{Laura Deckx}

The University of Queensland

\section{Shahid Ullah}

Flinders University

\section{Marjan Van den Akker}

Goethe-Universität Frankfurt am Main - Campus Bockenheim: Goethe-Universitat Frankfurt am Main

\section{Research Article}

Keywords: comorbidity, cancer, mortality, functional impairment, geriatric oncology

Posted Date: July 7th, 2021

DOI: https://doi.org/10.21203/rs.3.rs-605809/v1

License: (a) (i) This work is licensed under a Creative Commons Attribution 4.0 International License. Read Full License

Version of Record: A version of this preprint was published at Supportive Care in Cancer on September 22nd, 2021. See the published version at https://doi.org/10.1007/s00520-021-06567-1. 


\section{Abstract}

Purpose: To investigate if comorbidity predicts mortality and functional impairment in middle-aged individuals with cancer (50-64 years) as compared to older individuals.

Methods: A prospective cohort study. Outcomes were mortality and functional impairment at 5 years follow-up. Comorbidity was assessed using adjusted Charlson comorbidity index and polypharmacy ( $\geq 5$ drugs) as surrogate for comorbidity. Multivariate Cox-proportional hazards and binary logit models were used to assess the risk of 5-year mortality and functional impairment respectively.

Results: We included 477 middle-aged (50-64 years) and 563 older (65+ years) individuals with cancer. The prevalence of comorbidity (at least one disease in addition to cancer) was $29 \%$ for middle-aged and $45 \%$ for older individuals, with polypharmacy observed in $15 \%$ and $31 \%$ respectively. Presence of $\geq 3$ comorbidities nearly tripled the mortality risk in middle-aged individuals (HR 2.97, 95\% Cl: 1.43-6.16). In older individuals the HR was 1.7 (95\% Cl 1.1-2.8). Polypharmacy also significantly increased the risk for mortality in middle-aged (HR 2.35, 95\% Cl 1.32-4.16) but not in older individuals (HR 1.2, 95\% Cl 0.9-1.8). Polypharmacy quadrupled the risk for functional impairment in middle-aged (OR 4.0, 95\% $\mathrm{Cl} 1.59-10.06)$ and older individuals (OR 4.4, 95\% $\mathrm{Cl} 1.6-11.7$ ).

Conclusion: Comorbidity and polypharmacy are associated with inferior outcomes in younger and older cancer individuals with the strength of association in younger individuals exceeding that of older individuals. Assessment and management of comorbidity should be a priority for cancer care across all age groups.

\section{Background}

Comorbidity in people with cancer, defined as existence of condition(s) in addition to an index condition (cancer) impacts on cancer outcomes, including treatment toxicity, quality of life, function and survival.[1] Furthermore, both cancer itself and its treatment are associated with development of new chronic conditions and increase in severity of existing comorbid conditions, likely as a result of common risk factors and the direct effect of anticancer therapy.[2] Thus, assessing and addressing the additional burden of comorbid conditions is an important priority in cancer care.[3]

The management of patients with cancer and comorbidities is challenging. The decision-making process is more complex; not just because of the interaction of cancer and comorbidity and the impact on outcomes for both; but also because many individuals with cancer have more than one comorbidity and the management of one condition may adversely impact other conditions.[4] Clinical tools that account for the types of comorbid conditions, and their consequences, can assist with decision making.[5] While most of these tools are not yet routinely adopted into clinical practice, there is growing evidence that their application improves individuals' outcomes including quality of life and reduced treatment toxicity outcomes.[6] 
However, the evidence supporting the impact of these approaches seems to be confined to the population of patients with cancer who are older than 65 years of age and are managed under the auspices of geriatric oncology, with much less data pertaining to younger patients. The tools available to cancer clinicians have been developed and validated in older cancer individuals. For example, the CRASH (Chemotherapy Risk Assessment Scale) was constructed for individuals 70 years or older [7] and the Geriatric Oncology Assessment tool was validated in individuals 65 year or older.[8]

In contrast to ample data in older patients with cancer on the impact of comorbidities on functional decline and premature death,[9] there is less evidence on the prevalence and impact of comorbidities in middle-aged cancer individuals who may not fit the criteria for geriatric oncology management. This is an important gap in evidence for two reasons. Firstly, the data from general population indicates that while the proportion of individuals with comorbid conditions is highest among older adults, the absolute numbers of individuals with comorbid conditions are highest in those who are younger than 65 years.[10] Secondly, there is growing evidence that after cancer treatment, younger individuals with cancer carry mortality risks equivalent to much older individuals without cancer underscoring the need to explore the pattern and impact of comorbidity in individuals younger than 65 years.[11]

To address this gap, the present study investigated if middle-aged individuals with cancer (50-64 years) were vulnerable to the same negative outcomes as older individuals, including functional impairment, and premature death. We hypothesized that: (1) comorbidity was common in middle-aged individuals (50-64 years) with cancer, (2) poor functional status at baseline was common in middle-aged individuals with cancer, and (3) the presence of comorbidity at baseline was associated with poor outcomes (functional impairment, and mortality) in middle-aged individuals with cancer (50-64 years) with the strength of the association similar to older individuals ( $\geq 65$ years).

\section{Methods}

\section{Design and setting}

The study was the secondary analysis of an existing dataset of KLIMOP (Dutch acronym for a project on older cancer individuals in Belgium and the Netherlands) - an observational cohort study of individuals with cancer who were 50 years or older, recruited in 9 hospitals across Belgium and the Netherlands. The detailed study methodology has been described elsewhere.[12] Data were obtained from the hospital medical records and collected through personal interviews or self-administered questionnaires, at baseline (2010-2014) and after five years follow-up (2015-2019).

\section{Participants}

Individuals were eligible to participate if they were diagnosed with one of the following cancers: breast, prostate, lung or gastrointestinal, were aged 50 years or older, had life expectancy of more than 6 months, had a thorough command of Dutch, and were interviewed within 3 months of cancer diagnosis. 
Individuals were excluded if they were too ill to participate, had dementia or prior diagnosis of cancer, except non-melanoma skin cancer.

\section{Data items}

Data collection included socio-demographic information (sex, living arrangements, and educational level), medical information (e.g. cancer type, stage, comorbidities, number of medicines), and functional status.

Comorbidity was measured with an adjusted Charlson Comorbidity Index (CCI)[13] and was calculated with comorbidity data obtained from the medical records. Other comorbidities recorded in the medical record and not normally included in the $\mathrm{CCl}$ were included. These included: Parkinsons disease, blood transfusions, transplantation, thromboses, lung embolisms, pacemaker, angina pectoris, percutaneous transluminal coronary angioplasty or a coronary bypass).. Data was analysed according to the number of comorbidities $(0,1-2$, or $\geq 3$ ). Self-reported polypharmacy ( $\geq 5$ drugs) [14] was also examined as a surrogate for the presence of comorbidity.

Functional status was measured by Activities of Daily Living (ADL) using the Katz Index [15] and by Instrumental Activities of Daily Living (IADL) using the Lawton IADL-scale.[16] The Katz Index consists of six items (bathing, dressing, toileting, transferring, continence and feeding). The total sum score ranges from 0 (dependent) to 6 (independent). The Lawton IADL-scale consists of eight items in women (ability to use the telephone, shopping, cooking, housekeeping, doing laundry, taking own medication, transport, and ability to handle finances), and only five items in men, with cooking, housekeeping and laundry excluded. Functional impairment was defined as dependence on at least one domain of ADL or IADL.

\section{Statistical analyses}

All statistical analyses were performed using Stata statistical software, version 16.1. Patient's characteristics were expressed as median and intra quartile range (IQR) for continuous skewed data and proportions were presented as percentages of the respective denominator. Mann-Whitney U-test and standard Chi-square test for association with continuity correction, where appropriate, were used to explore the significant differences of individuals' characteristics between middle-aged (50-64 years) and older ( $\geq 65$ years) people with cancer.

Survival was measured from the date of cancer diagnosis to the date of death, individuals were censored at date of loss to follow-up or census date. The census date was assigned at 5 years follow-up for each individual patient. Date of death was recorded using data from medical records, obituaries, or contacting relatives.

Cox proportional hazard models were applied to examine the survival outcomes between two cohorts. Univariate models were first performed to explore the association between each covariate and the risk of mortality. Multivariate modelling was then undertaken by adding covariates considered clinically important and statistically significant from the univariate models, in order to adjust for confounding between variables. The variables included in the multivariate model were age, gender, and education. The 
estimates were calculated using the likelihood ratio method and were expressed as hazard ratios (HRs). Proportional hazard assumption was tested by log-log plot of survival and Schoenfeld Residuals. Survival curves between cohorts were evaluated by standard Kaplan-Meier survival curves and groups were compared by log-rank test. The departure from linearity for continuous predictors was tested by linear spline for the adaptive model and found no evidence of non-linearity. The risk of death within 5 years were established from the Cox proportion hazard models by restricting the predictive model to 5 years death or the time of last follow-up.

A logistic regression model was applied to examine the functional impairment between two cohorts. The functional impairment was treated as a binary outcome at 5-year follow-up time. The same strategy from Cox proportional hazard model was applied for univariate and multivariate models. The estimates were calculated using the likelihood ratio method and were expressed as odds ratios (ORs) from the logit model. The ORs were considered statistically significant if their $95 \% \mathrm{Cl}$ did not include unity. The more the OR deviated from 1, the stronger the association between the exposure variable and outcome. Model diagnostics and goodness of fit were evaluated by Receiver Operator Characteristic (ROC) curves and Cstatistics.

The two-sided test was performed for all analysis and the level of significance was set at $p<0.05$.

\section{Ethics}

The Ethical Review Board of KU Leuven and UZ Leuven (S52097 - ML6279) and the Maastricht University Medical Centre (NL31414.068.10) approved the study protocol. The study was conducted in compliance with Good Clinical Practice guidelines Procedures, the principles of the Declaration of Helsinki (version October 2008) and the Belgian (law of 7 may 2004 concerning clinical trials with humans) and Dutch (Medical Research Involving Human Subjects Act and Personal Data Protection Act) laws. All individuals signed informed consent.

\section{Results}

A total of 1040 individuals with cancer aged 50 to 93 were included in the study with $477(45.9 \%)$ in the middle age group (50-64) and remainder in the older group (Table 1). The majority were women $(n=710$, $68 \%)$, living together with someone $(n=779,75 \%)$, either with partner, children, or both. The majority of older individuals ( $\geq 65$ years) left school before they were $18(n=335,60 \%)$, whereas the majority of middle-aged individuals ( $n=291,50-64$ years) left school after the age of 18 . The most common cancer types were prostate $(n=472,45 \%)$, breast $(n=372,36 \%)$, and colorectal cancer $(n=148,14 \%)$. When comparing the middle-age and the older cohort, the older cohort had slightly lower proportion of women, were more likely to live alone or in an institution (such as nursing home) and were more likely to complete education earlier. While there was no difference in the distribution of cancer types among the two cohorts, the older group was less likely to be diagnosed with earlier stages of cancer. The prevalence of comorbidity was $29 \%$ for middle-aged and $45 \%$ for older individuals with higher proportion of older 
individuals having 3 or more comorbidities $(n=61 ; 10.8 \%$ versus $n=16 ; 3.4 \%)$. Polypharmacy was also more common in older individuals (172 (31\%) vs 70 (15\%) (Table 2$)$. 
Table 1

Participants characteristics $(n=1040)$.

\begin{tabular}{|c|c|c|c|c|c|}
\hline & \multirow{2}{*}{\multicolumn{2}{|c|}{$\begin{array}{l}\text { Middle-aged } \\
\text { (50-64 years) } \\
\mathrm{N}=477(45.9 \%)\end{array}$}} & \multirow{2}{*}{\multicolumn{2}{|c|}{$\begin{array}{l}\text { Older } \\
(65+\text { years }) \\
N=563(54.1 \%)\end{array}$}} & \multirow[b]{3}{*}{ P-value } \\
\hline & & & & & \\
\hline & $\mathbf{N}$ & $\%$ & $\mathbf{N}$ & $\%$ & \\
\hline \multicolumn{6}{|c|}{ SOCIO-DEMOGRAPHIC CHARACTERISTICS } \\
\hline Age (years): median \pm IQR & 58 & $(54-61)$ & 73 & $(68-78)$ & $<0.001$ \\
\hline Gender & & & & & $<0.01$ \\
\hline Male & 131 & 27.5 & 199 & 35.3 & \\
\hline Female & 346 & 72.5 & 364 & 64.7 & \\
\hline Living circumstances & & & & & $<0.001$ \\
\hline Alone & 76 & 15.9 & 149 & 26.5 & \\
\hline With someone & 391 & 82.0 & 388 & 68.9 & \\
\hline Institutionalized & - & - & 15 & 2.7 & \\
\hline Other & 6 & 1.3 & 11 & 2.0 & \\
\hline Missing & 4 & 0.8 & - & - & \\
\hline Age at leaving school & & & & & $<0.001$ \\
\hline$<18$ years & 173 & 36.3 & 335 & 59.5 & \\
\hline $18+$ years & 291 & 61.0 & 214 & 38.0 & \\
\hline Missing & 13 & 2.7 & 14 & 2.5 & \\
\hline \multicolumn{6}{|c|}{ CLINICAL CHARACTERISTICS } \\
\hline Cancer stage & & & & & $<0.01$ \\
\hline Stage I-III & 367 & 76.9 & 356 & 63.2 & \\
\hline Stage IV & 13 & 2.7 & 30 & 5.3 & \\
\hline Missing & 97 & 20.3 & 177 & 31.4 & \\
\hline Cancer type & & & & & 0.21 \\
\hline Breast & 178 & 37.3 & 194 & 34.5 & \\
\hline Prostate & 210 & 44.0 & 262 & 46.5 & \\
\hline
\end{tabular}




\begin{tabular}{|c|c|c|c|c|c|}
\hline & \multirow{2}{*}{\multicolumn{2}{|c|}{$\begin{array}{l}\text { Middle-aged } \\
\text { (50-64 years) } \\
\mathrm{N}=477(45.9 \%)\end{array}$}} & \multirow{2}{*}{\multicolumn{2}{|c|}{$\begin{array}{l}\text { Older } \\
(65+\text { years }) \\
N=563(54.1 \%)\end{array}$}} & \multirow[b]{3}{*}{ P-value } \\
\hline & & & & & \\
\hline & $\mathbf{N}$ & $\%$ & $\mathbf{N}$ & $\%$ & \\
\hline Colorectal & 62 & 13.0 & 86 & 15.3 & \\
\hline Lung & 10 & 2.1 & 15 & 2.7 & \\
\hline Liver & 4 & 0.8 & 1 & 0.2 & \\
\hline Oesophagus & 5 & 1.0 & 2 & 0.4 & \\
\hline Pancreas & 3 & 0.6 & 1 & 0.2 & \\
\hline Stomach/Intestine & 3 & 1.0 & 2 & 0.4 & \\
\hline
\end{tabular}


Table 2

Prevalence of 10 most common comorbidities at baseline $(n=1040)$.

\begin{tabular}{|c|c|c|c|c|c|}
\hline & \multirow{2}{*}{\multicolumn{2}{|c|}{$\begin{array}{l}\text { Middle-aged (50-64 years) } \\
\mathrm{N}=477(45.9 \%)\end{array}$}} & \multirow{2}{*}{\multicolumn{2}{|c|}{$\begin{array}{l}\text { Older (65 + years) } \\
\mathrm{N}=563(53.9 \%)\end{array}$}} & \multirow[b]{3}{*}{ P-value } \\
\hline & & & & & \\
\hline & $\mathbf{N}$ & $\%$ & $\mathbf{N}$ & $\%$ & \\
\hline Comorbidity & & & & & $<0.001$ \\
\hline No & 338 & 70.9 & 310 & 55.1 & \\
\hline Yes & 139 & 29.1 & 253 & 44.9 & \\
\hline No of comorbidities* & & & & & $<0.001$ \\
\hline 0 & 338 & 70.9 & 310 & 55.1 & \\
\hline $1-2$ & 123 & 25.8 & 192 & 34.1 & \\
\hline $3+$ & 16 & 3.4 & 61 & 10.8 & \\
\hline Polypharmacy (number of drugs $\geq 5$ ) & & & & & $<0.001$ \\
\hline No & 407 & 85.3 & 391 & 69.4 & \\
\hline Yes & 70 & 14.7 & 172 & 30.6 & \\
\hline \multicolumn{6}{|l|}{ Type of comorbidities } \\
\hline Connective tissue disease & 33 & 6.9 & 48 & 8.5 & 0.25 \\
\hline Diabetes mellitus & 28 & 5.9 & 64 & 11.4 & $<0.001$ \\
\hline Chronic pulmonary disease & 26 & 5.5 & 53 & 9.4 & 0.01 \\
\hline Heart Failure & 14 & 2.9 & 37 & 6.6 & $<0.01$ \\
\hline Angina pectoris & 12 & 2.9 & 22 & 5.6 & 0.01 \\
\hline Peripheral vascular disease & 11 & 2.3 & 26 & 4.6 & 0.04 \\
\hline Myocardial infarction & 10 & 2.1 & 35 & 6.2 & $<0.001$ \\
\hline Cerebrovascular disease & 8 & 1.7 & 27 & 4.8 & $<0.01$ \\
\hline Renal Disease & 6 & 1.3 & 15 & 2.7 & 0.09 \\
\hline Peptic ulcer disease & 4 & 0.8 & 18 & 3.2 & $<0.01$ \\
\hline
\end{tabular}

The median follow-up time was 4.9 years for middle-aged individuals and 3.9 years for older individuals. Five years after cancer diagnosis, the majority $(n=265 ; 55 \%)$ of middle-aged cancer individuals were 
alive, 64 (13\%) were deceased and $148(31 \%)$ were lost to follow-up. In the older cohort, 38\% $(n=211)$ were still alive, 30\% ( $n=170)$ were deceased and $182(32 \%)$ were lost to follow-up. The impact of comorbidity on mortality is summarized in Fig. 1. Presence of anycomorbidity did not significantly predict mortality in either cohort (in middle age cohort $\mathrm{HR}=1.53(95 \% \mathrm{Cl} 0.86-2.73) \mathrm{p}=0.15 \mathrm{C}=0.75$, in older cohort HR $=1.35(95 \% \mathrm{Cl} 0.95-1.90) p=0.09 \mathrm{C}=0.63)$. However, presence of $\geq 3$ comorbidities was associated with nearly triple the risk for mortality in middle-aged individuals (HR 2.97, 95\% Cl: 1.43-6.16 $p<0.01)$. In older individuals the HR for mortality if $\geq 3$ comorbidities present was $1.71(95 \% \mathrm{Cl} 1.05-$ $2.78 p=0.03$ ). Polypharmacy was also significantly associated with the increased the risk for mortality in middle-aged (HR 2.35, 95\% Cl 1.32-4.16 p < 0.01) but not in older individuals (HR 1.24, 95\% Cl 0.87-1.77, $p=0.23)$.

The impact of comorbidity on functional impairment is summarized in Fig. 2. Comorbidity predicted functional impairment in older adults only $(\mathrm{OR}=2.44(95 \% \mathrm{Cl} 1.22-4.89) \mathrm{p}=0.01)$. Polypharmacy quadrupled the risk for functional impairment in middle-aged (OR 4.00, 95\% $\mathrm{Cl} 1.59-10.06 \mathrm{p}<0.01$ ) as well as older individuals (OR 4.36, 95\% $\mathrm{Cl} 1.63-11.68, \mathrm{p}<0.01)$.

Figure 3 summarises changes to functional status at 5 year follow-up according to baseline status. At baseline, 209 (44\%) of middle-aged individuals and $339(60 \%)$ of older individuals had functional impairment. While $26 \%$ and $20 \%$ of middle aged and older individuals who were independent at baseline maintained their independent status, $17 \%$ of dependent middle age individuals and less than $1 \%$ of older dependent individuals recovered their functional independence at 5 year of follow-up.

\section{Discussion}

This prospective cohort study of middle-aged and older individuals with cancer shows that comorbidities are common in individuals with cancer; with nearly a third of middle-aged individuals and nearly half of older adults with cancer reporting comorbidities. Those with 3 comorbidities or more were at higher risk of mortality, and comorbidity was associated with functional impairment in older but not middle-aged individuals.

The prevalence and types of comorbidities reported in this study was similar to that reported in other studies of individuals with cancer from UK, Europe and New Zealand [17-19] reflecting high prevalence of comorbidities in the general population that is not confined to just older individuals.[20] In fact, the prevalence of comorbid disease in the older cohort in the present study reflects those reported in cancer studies but is lower than what is reported in the general population study for the similar age group which shows prevalence or more than one chronic condition of $64.9 \%$ in those 65-84.[20] This finding may suggest that older patients with cancer may be preselected for cancer treatment and those with many comorbidities may not be treated or followed up for cancer.

The unique contribution of the present study is the analysis of the impact of comorbidities on mortality and functional status. Our data suggests that the impact of comorbidity on mortality is greater in middle age than in older individuals. We are unable to determine the reason for this finding. It may reflect a 
selection bias with older individuals who have survived with comorbidities possibly being fitter and better adapted to life with comorbidities or underreporting their comorbidities. Similarly, polypharmacy which could serve as a surrogate for comorbidities was a stronger predictor of mortality in middle-aged, more than in older individuals. Given that data on medications was more likely to be accurately extracted from the hospital records, it is less likely that this finding reflects the selection bias or under-reporting but rather than in older individuals, the impact of comorbidity on mortality is likely to diluted by the expected age related mortality much more so than in younger individuals.

Polypharmacy but not comorbidity predicted functional impairment perhaps suggesting that polypharmacy is a more sensitive indicator of severe disease. This observation highlights the importance of selecting the optimal tool to assess comorbidity - simply counting comorbid conditions may not be the most sensitive way of identifying those with the highest disease burden. Surrogate markers like assessment of polypharmacy may be more useful. Research into optimal comorbidity assessment tools including function assessment and biomarkers is needed. [21]

In our cohort the pattern of change of functional impairment showed that some individuals recovered but the recovery was more likely in the middle-aged individuals than older individuals. Further research is needed as to why this is so. The faster recovery may reflect the different biology of functional impairment in different age groups or the health system issues where older patients who have functional decline are not offered care to assist them in recovery.

Our findings suggesting that comorbidity and its surrogate, polypharmacy, may be more important than age alone in identifying individuals with cancer at risk of poor outcomes. These findings have important implication for practice of oncology underscoring the importance of assessment of comorbidity and function in older and younger individuals with cancer, similarly to what is increasingly recommended in the general population.[10] Special attention should be paid to those individuals who have more than one comorbid condition and coinciding functional impairment. While the emergence of geriatric oncology as a discipline, facilitates care delivery for the older individuals with cancer and comorbid conditions, there are currently no provisions for similar approaches for younger individuals. As research suggests that cancer and its treatment are associated with premature ageing, there is also a need for a more fundamental research into the understanding of multimorbidity in cancer and its aetiology especially in younger individuals.

The strength of this study lies in its large size, prospective nature and long follow-up. Its limitations relate to missing data and using hospital records for data sources which may be incomplete. Some subgriyos included only small numbers of individuals (for example there were only few individuals with comorbidities who had no functional impairment) leading to wide confidence intervals for some of the analyses.

In conclusion, this study of middle aged and older adults with cancer shows that comorbid disease is common in younger and older individuals with cancer and is associated with higher risk of mortality and functional impairment. There is a need for research into comorbidities in younger individuals with cancer 
and for the development of tools to assess and manage comorbid conditions in all individuals with cancer, not just those who are older.

\section{Declarations}

Funding: This study is funded by the European Union / Interreg IV Grensregio Vlaanderen - Nederland (IVA-VLANED-3.46) and Kom op tegen Kanker (Stand up to Cancer - the Flemish cancer league) (10482))

Conflicts of interest/Competing interests Laura Deckx is currently employed by Kom op tegen Kanker who partially funded this study. However, at the time of the data collection Laura was not employed at Kom op tegen Kanker and was not the investigator on the original grant.

Availability of data and material Data are available on reasonable request

Code availability (software application or custom code) Code is available upon request

Authors' contributions: all authors contributed to the conceptualization, formal analysis, methodology, and writing.

Ethics approval: The Ethical Review Board of KU Leuven and UZ Leuven (S52097 - ML6279) and the Maastricht University Medical Centre (NL31414.068.10) approved the study protocol.

Consent to participate: All individuals signed informed consent.

Consent to publish: All authors granted the consent to publish the manuscript.

\section{References}

1. Sarfati D, Koczwara B, Jackson C (2016) The impact of comorbidity on cancer and its treatment. CA Cancer J Clin 66(4):337-350. doi:10.3322/caac.21342

2. Ng HS, Koczwara B, Roder D, Vitry A (2018) Changes in the prevalence of comorbidity in the Australian population with cancer, 2007-2014. Cancer Epidemiol 54:56-62.

doi:10.1016/j.canep.2018.03.010

3. Ritchie CS, Kvale E, Fisch MJ (2011) Multimorbidity: An Issue of Growing Importance for Oncologists. Journal of Oncology Practice 7(6):371-374. doi:10.1200/JOP.2011.000460

4. Jack E, Maskrey N, Byng R (2018) SHERPA: a new model for clinical decision making in patients with multimorbidity. The Lancet: 392:1397-1399. doi:10.1016/S0140-6736(18)31371-0

5. Webster S, Lawn S, Chan R et al (2020) The role of comorbidity assessment in guiding treatment decision-making for women with early breast cancer: a systematic literature review. Support Care Cancer 28:1041-1050. doi:10.1007/s00520-019-05218-w

6. Li D, Sun C-L, Kim H, Chung V, Koczywas M, Fakih M et al (2020) Geriatric assessment-driven intervention (GAIN) on chemotherapy toxicity in older adults with cancer: A randomized controlled 
trial. Journal of Clinical Oncology 38(15_suppl):12010- doi. 10.1200/JC0.2020.38.15_suppl.12010

7. Extermann M, Boler I, Reich R, Lyman GH, Brown RH, DeFelice J et al (2010) The Chemotherapy Risk Assessment Scale for High-Age Patients (CRASH) score: Design and validation. J Clin Oncol 28(15_suppl):9000-. doi:10.1200/jco.2010.28.15_suppl.9000

8. Wildiers H, Heeren P, Puts M, Topinkova E, Janssen-Heijnen MLG, Extermann M et al (2014) International Society of Geriatric Oncology Consensus on Geriatric Assessment in Older Patients With Cancer. Journal of Clinical Oncology 32(24):2595-2603. doi:10.1200/JC0.2013.54.8347

9. Yancik R, Wesley MN, Ries LA, Havlik RJ, Edwards BK, Yates JW (2001) Effect of age and comorbidity in postmenopausal breast cancer patients aged 55 years and older. JAMA. Feb 21;285(7):885 - 92. doi: 10.1001/jama.285.7.885

10. Hanlon P, Nicholl BI, Jani BD et al (2018) Frailty and pre-frailty in middle-aged and older adults and its association with multimorbidity and mortality: a prospective analysis of 493737 UK Biobank participants. Lancet Public Health 3:e323-e332. doi:10.1016/S2468-2667(18)30091-4

11. de Vries S, Schaapveld M, Janus CPM et al (2021) Long-Term Cause-Specific Mortality in Hodgkin Lymphoma Patients, JNCl. J Natl Cancer Inst 113(6):760-769. doi:10.1093/jnci/djaa194

12. Deckx L, van Abbema D, Nelissen K et al (2011) Study protocol of KLIMOP: a cohort study on the wellbeing of older cancer patients in Belgium and the Netherlands. BMC Public Health 11:825. doi:10.1186/1471-2458-11-825

13. Charlson ME, Pompei P, Ales KL, MacKenzie CR (1987) A new method of classification of prognostic comorbidity for longitudinal studies: development and validation. J Chron Disease 40(5):373-383. doi:10.1016/0021-9681(87)90171-8

14. Masnoon N, Shakib S, Kalisch-Ellett L, Caughey GE (2017) What is polypharmacy? A systematic review of definitions. BMC Geriatr 10(1):230. doi:10.1186/s12877-017-0621-2 17 ) .

15. Katz S (1983) Assessing self-maintenance: Activities of daily living, mobility and instrumental activities of daily living. JAGS 31(12):721-726. doi:10.1111/j.1532-5415.1983.tb03391.x

16. Lawton MP, Brody EM (1969) Assessment of older people: Self-maintaining and instrumental activities of daily living. Gerontologist 9(3):179-186

17. Fowler $\mathrm{H}$, Belot $A$, Ellis $L$ et al (2020) Comorbidity prevalence among cancer patients: a populationbased cohort study of four cancers. BMC Cancer 20:2. doi:10.1186/s12885-019-6472-9

18. Luque-Fernandez MA, Redondo Sánchez D, Rodriguez-Barranco M, CarmonaGarcia MC, MarcosGragera R, Sanchez-Perez MJ (2020) Multimorbidity by patient and tumor factors and time-tosurgery among colorectal cancer patients in Spain: a population-based study. Clinical Epidemiology 12:31-40 20. doi:10.2147/CLEP.S229935

19. Sarfati D, Gurney J, Lim BT, Bagheri N, Simpson A, Koea J et al (2016) Identifying important comorbidity among cancer populations using administrative data: prevalence and impact on survival. Asia Pac J Clin Oncol 12(1):e47-e56. doi:10.1111/ajco.12130

20. Barnett K, Mercer SW, Norbury M, Watt G, Wyke S, Guthrie B (2012) Epidemiology of multimorbidity and implications for health care, research, and medical education: a cross-sectional study. Lancet 
380:37-43. doi:10.1016/S0140-6736(12)60240-2

21. Overcash J, Cope DG, Van Cleave JH (2018) Frailty in Older Adults: Assessment, Support, and Treatment Implications in Patients With Cancer Clin J Oncol Nurs.2018 22(6):8-18. doi:

10.1188/18.CJON.S2.8-18. doi: 10.1188/18.CJON.S2.8-18

\section{Figures}

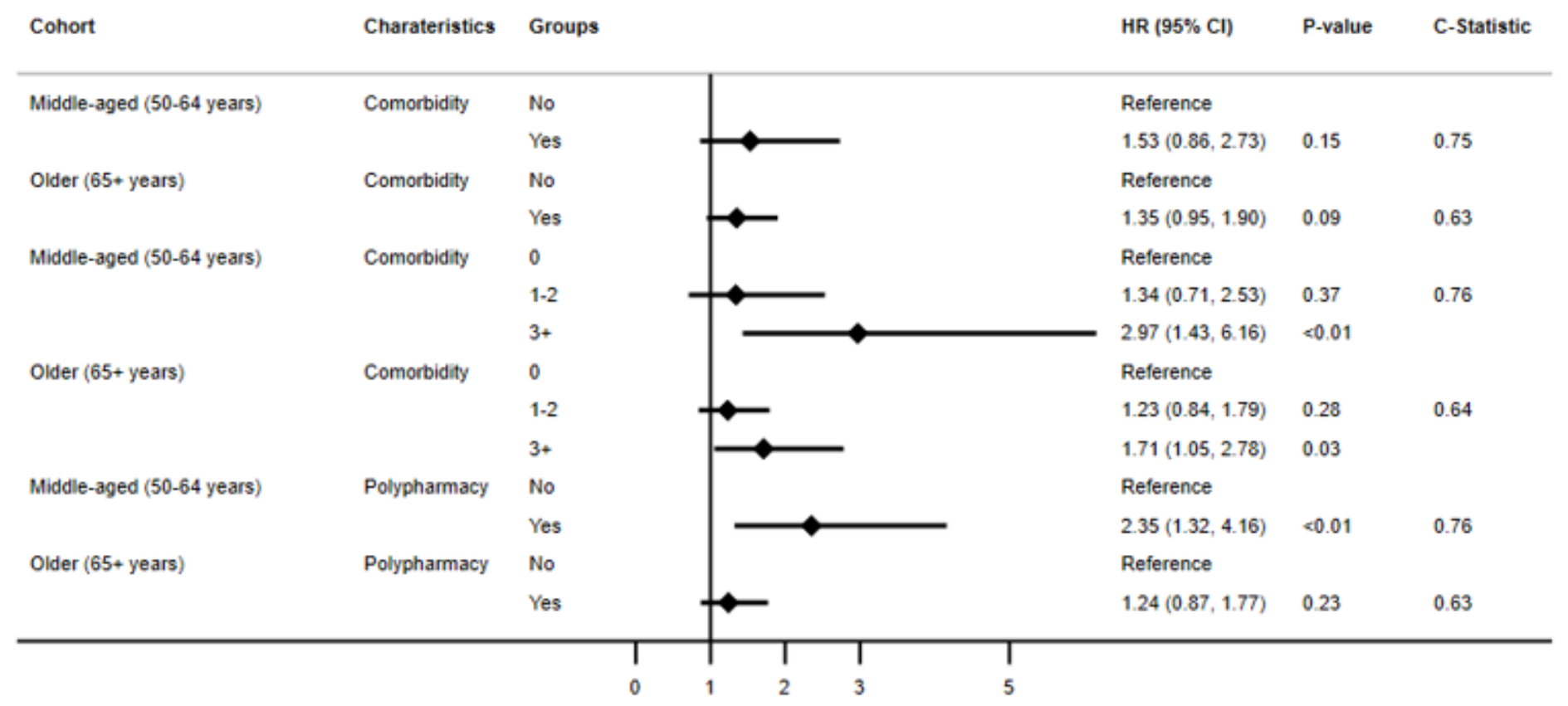

\section{Figure 1}

The impact of comorbidity on mortality. 


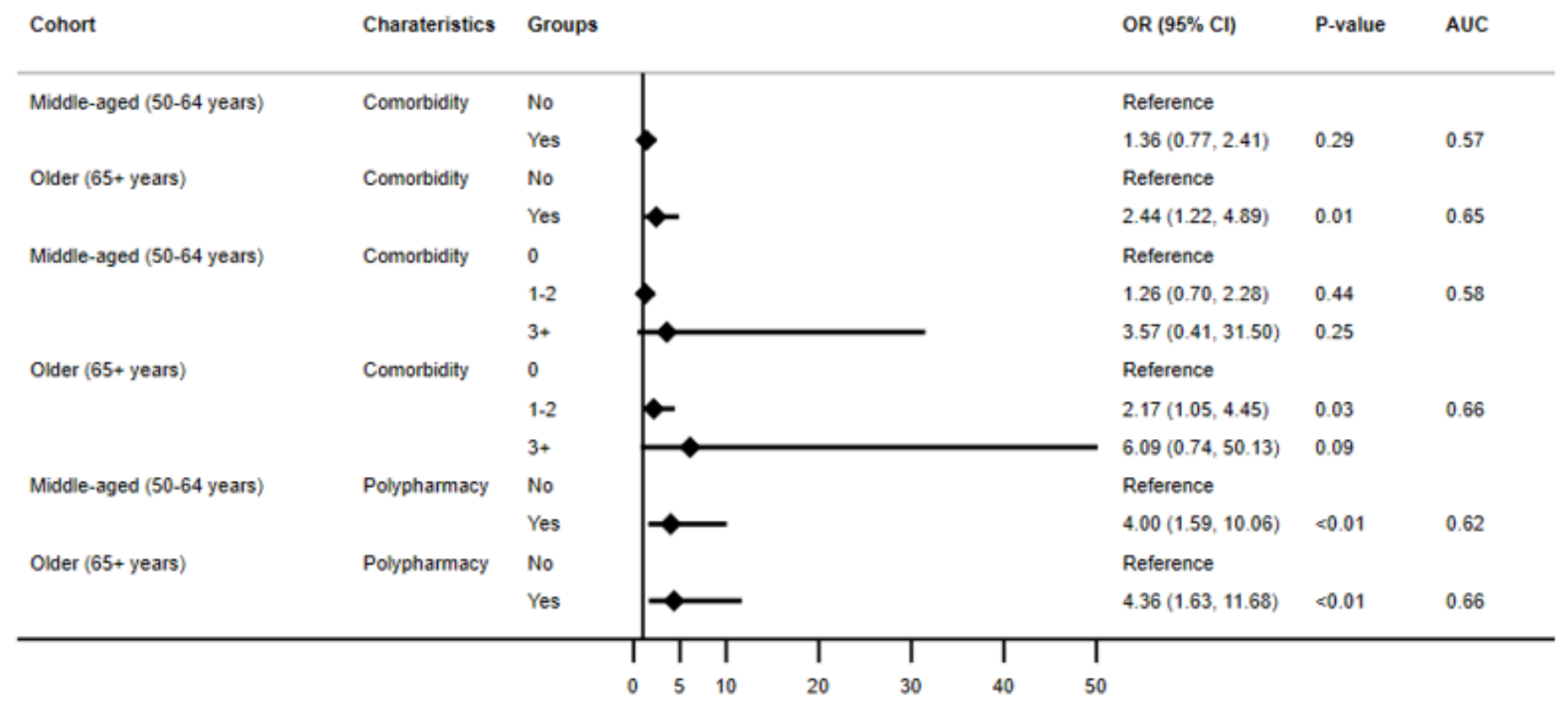

\section{AUC - Area under curve}

\section{Figure 2}

The impact of comorbidity on functional impairment. 
$50 \%$

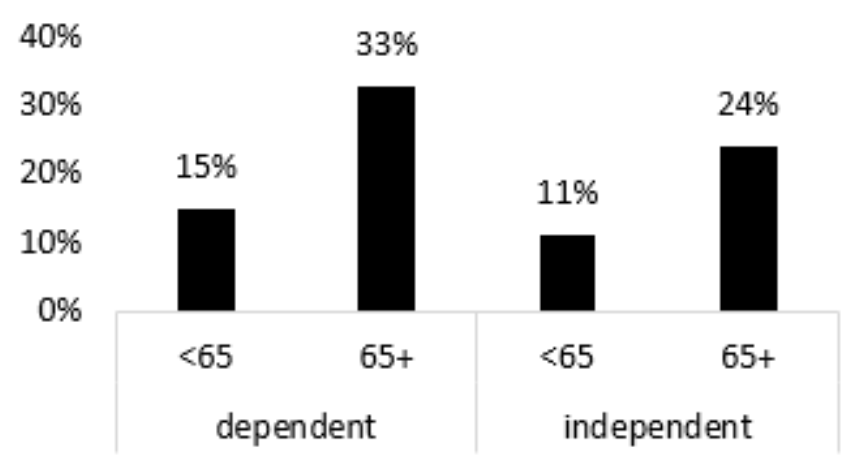

\% functional status improved or deteriorated

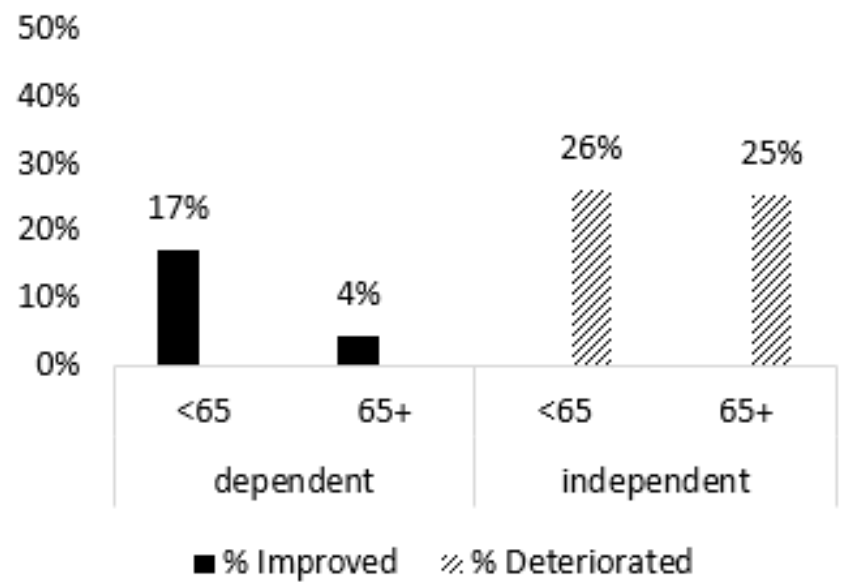

$50 \%$

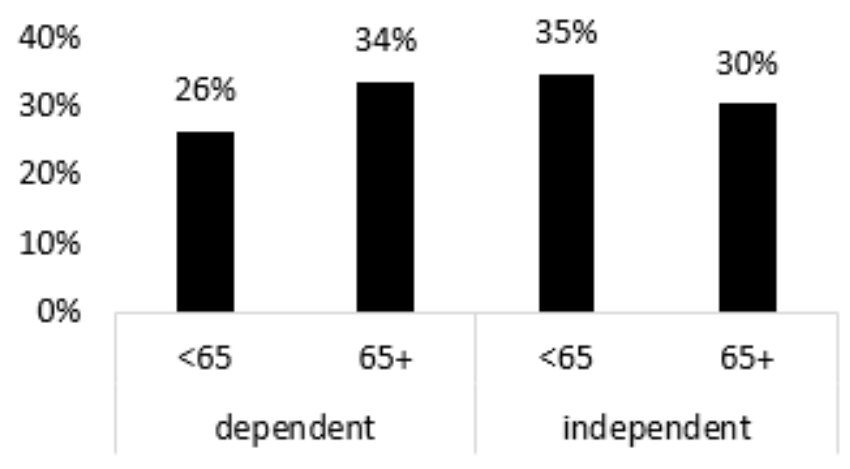

$\%$ who remained dependent or independent

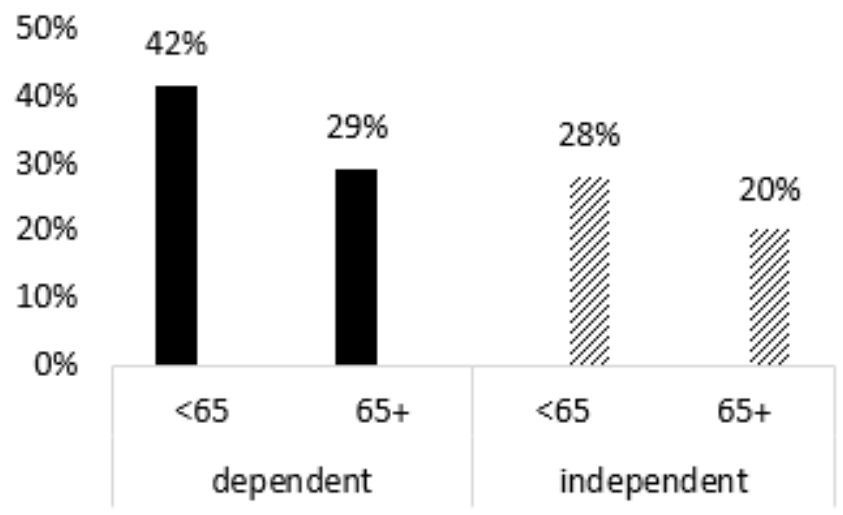

- $\%$ remained dependent $\% \%$ remained independent

\section{Figure 3}

Changes in functional status according to baseline status. The denominator is the number of individuals who were dependent $(n<65=209 ; n \geq 65=339)$ or independent $(n<65=268 ; n \geq 65=224)$ at baseline 